\title{
INDUCCIÓN DE CRAVING POR COMIDA MEDIANTE REALIDAD VIRTUAL NO INMERSIVA
}

\author{
Mario Agliaro-López, Marta Ferrer-Garcia, Joana Pla-Sanjuanelo \\ Y JosÉ GuTIÉRREZ-MALDONADO
}

Facultad de Psicología, Universidad de Barcelona, Barcelona, España

\begin{abstract}
Resumen: La terapia de exposición a señales se ha propuesto como un tratamiento eficaz para las conductas de atracón al propiciar la extinción de la asociación entre los estímulos relacionados con la ingesta compulsiva y la respuesta de craving. El objetivo de este estudio es evaluar si la exposición a alimentos en entornos de realidad virtual (RV) es capaz de producir niveles de craving consistentes con diferencias individuales en craving-rasgo y craving-estado, de manera que puedan ser utilizados en programas de tratamiento basados en exposición a señales. Los participantes fueron expuestos a 10 alimentos en cuatro entornos virtuales. Las personas que presentaban mayores niveles de craving-rasgo y cravig-estado mostraron mayores deseos de comer durante la exposición a los entornos virtuales. Estos resultados constituyen un indicador de validez del uso de procedimientos basados en la exposición virtual a comida para llevar a cabo intervenciones basadas en técnicas de exposición a señales.
\end{abstract}

Palabras clave: Craving; exposición a señales; realidad virtual; bulimia nerviosa; trastorno por atracón.

\section{Inducing food craving by means of non immersive virtual reality}

\begin{abstract}
The binge behavior has been associated with elevated levels of food craving. Cue exposure therapy has been proposed as an effective treatment for binge behavior, facilitating the extinction of the association between the stimuli associated with binge eating and the craving response. The aim of this study is to evaluate whether exposure to food in virtual environments can produce craving levels consistent with individual differences in trait-craving and state-craving, such that they can be used in treatment programs based on cue exposure. The participants were exposed to 10 foods in four virtual environments using non immersive devices. Consistent with the predictions, individuals who had higher levels of trait-craving and state-craving showed a greater desire to eat during exposure to different virtual environments. These results are indicative of the validity of procedures based on food exposure in virtual environments to carry out interventions based on cue exposure techniques.
\end{abstract}

Keywords: Craving; cue exposure; virtual reality; bulimia nervosa; binge eating disorder.

\section{INTRODUCCIÓN}

Los trastornos de la conducta alimentaria (TCA) se caracterizan por alteraciones de la in-

\footnotetext{
Recibido: 9 junio 2014; aceptado: 29 junio 2014.

Correspondencia: José Gutiérrez-Maldonado, Facultad de Psicología, Departamento de personalidad, evaluación y tratamientos psicológicos, Universidad de Barcelona. Paseo Valle de Hebrón, 171, 08035 Barcelona, España. Correo-e: jgutierrezm@ub.edu

Agradecimientos: Este estudio ha sido realizado gracias a la financiación del Ministerio de Ciencia e Innovación (Proyecto PSI2011-28801: "Exposición a señales mediante realidad virtual para el tratamiento de la bulimia nerviosa").
}

gesta que, ya sea por exceso o por defecto, conllevan importantes cambios en el peso y/o graves perturbaciones en la relación que se establece con la comida. Como consecuencia, aparecen importantes problemas físicos y del funcionamiento psicosocial, siendo además frecuentes las recaídas. La bulimia nerviosa $(\mathrm{BN})$ y el trastorno por atracón (TA), ya incluido en el DSM-5 (APA, 2013), comparten la presencia de episodios recurrentes de atracón que se experimentan con sensación de falta de control. En el síndrome bulímico, estos episodios de ingesta voraz se siguen de conductas compensatorias cuyo obje- 
tivo principal es la eliminación de las calorías ingeridas y la disminución del sentimiento de culpa generado por dicho comportamiento. En el trastorno por atracón, por el contrario, no se ponen en marcha mecanismos compensatorios para evitar la ganancia de peso.

Procesos de condicionamiento clásico e instrumental determinan en gran medida qué alimentos, cuándo, con qué frecuencia y qué cantidad se consume, y determinan las respuestas de evitación y de búsqueda de recompensa (Koskina, Campbell, y Schmidt, 2013). Estos mismos procesos parecen jugar un papel importante en la aparición y mantenimiento de la conducta de atracón. Jansen (1994, 1998; Nederkoon y Jansen, 2002) propone un modelo basado en los principios del condicionamiento clásico para explicar el comportamiento de atracón presente en pacientes con BN. Según este modelo, la ingesta excesiva de alimentos se considera el estímulo no condicionado, mientras que todos los estímulos asociados con este comportamiento de atracón constituyen los estímulos condicionados (por ejemplo, el tipo de comida, el lugar donde se lleva a cabo o el momento del día en que se realiza el atracón). La exposición a los estímulos condicionados provoca respuestas fisiológicas y bioquímicas que se experimentan subjetivamente como un deseo intenso de comer (craving), que lleva a la ingesta excesiva de alimentos. El craving es definido como un estado motivacional que promueve la búsqueda de comida y la consiguiente realización de conductas de ingesta (Cepeda-Benito, Gleaves, Willians, y Erath, 2000). Estudios previos muestran que las personas que llevan a cabo atracones o que experimentan pérdida de control sobre la ingesta son las que presentan más craving, evaluado como característica estable y consistente, es decir como rasgo (Gómez, Yela, Salgado, y Cortés, 2011).

La terapia de exposición a señales busca extinguir la respuesta de craving al romper la asociación entre los estímulos condicionados y el estímulo incondicionado (ingesta). En la terapia de exposición a señales, los pacientes son expuestos a aquellos alimentos que suelen ingerir durante los episodios de atracón. Durante la exposición pueden tocarlos, olerlos, manipularlos y actuar como si fueran a ingerir el ali- mento, pero se evita que lleven a cabo el atracón (prevención de respuesta). Estudios previos aportan evidencia sobre la eficacia de la terapia de exposición a señales para el tratamiento de la conducta de atracón en pacientes con BN (Jansen, Broekmate, y Heymans, 1992; Jansen, van den Hout, de Loof, Zandbergen, y Griez, 1989; Martínez-Mayén et al., 2007; Toro et al., 2003). Los resultados de estos trabajos muestran importantes mejoras en la sintomatología, eliminándose o reduciéndose de manera significativa las conductas de purga y atracón y mejorándose el estado general del paciente. Sin embargo, los estudios publicados son escasos y cuentan con muestras muy pequeñas por lo que estos resultados deben analizarse con cautela (Ferrer-Garcia, Gutiérrez-Maldonado, y Riva, 2013; Koskina et al., 2013).

En los estudios en que se ha empleado la terapia de exposición a señales para el tratamiento de la BN se ha empleado la exposición en vivo, ya fuese en consulta (Martínez-Mayén et al., 2007; Toro et al., 2003) o en la situación real en que la persona suele llevar a cabo la conducta de atracón (Jansen et al., 1992; Jansen et al., 1989; Schmidt y Marks,1988). Aun contando con claras ventajas, la exposición en vivo tiene ciertas limitaciones que deben tenerse en cuenta (Gutiérrez-Maldonado, Ferrer-Garcia, Caqueo-Urízar, y Moreno, 2010). En aquellos casos en que la exposición en vivo se lleva a cabo en la consulta, el terapeuta debe disponer de los alimentos necesarios para llevarla a cabo. Además, sólo permite la exposición a estímulos proximales (alimentos), lo que reduce la capacidad de generalización a entornos diferentes a la consulta. Por otro lado, la exposición en la situación real en que el paciente suele realizar la conducta de atracón implica que el terapeuta tenga que desplazarse. Además, existe el riesgo de que los pacientes rechacen el contacto directo con el estímulo. La alternativa más comúnmente empleada, la exposición en imaginación, también presenta ciertos inconvenientes. Las limitaciones más importantes son la falta de control sobre lo que los participantes están imaginando y las dificultades que tienen muchas personas para visualizar con suficiente grado de realismo una situación. Existe una tercera opción en los procedimientos de exposición que 
ha ido ganando terreno en los últimos años: la realidad virtual.

La realidad virtual (RV) se define como aquella tecnología informática que genera entornos tridimensionales con los que el usuario interactúa en tiempo real, produciendo una sensación de inmersión semejante a la presencia en el mundo real (Gutiérrez-Maldonado, 2002; García-Rodríguez, Pericot-Valverde, Gutiérrez-Maldonado, y Ferrer-García, 2009). Autores como Gorini y Riva (2008) la definen como un entorno simulado por ordenador con el cual la persona puede interactuar. Entre las ventajas que ofrece esta tecnología destacan que: no se compromete la privacidad del paciente; se percibe como un contexto seguro, por lo que es más fácilmente aceptada; permite un elevado control sobre las variables; permite recrear situaciones más vívidas que en la exposición imaginada; permite al terapeuta saber qué es lo que pasa exactamente en la situación; es un procedimiento adecuado para las personas con poca capacidad imaginativa e incluso permite recrear situaciones imposibles o improbables (Botella, García-Palacios, Baños y Quero, 2007).

En un estudio realizado por Pallavicini et al. (2013) sobre la efectividad de los tratamientos que hacen uso de RV se muestra que sin un importante grado de presencia las ventajas que ofrece la RV desaparecen y la inducción de respuesta emocional es incluso inferior a la que proporcionan otros medios más económicos. La medida en que una persona se siente presente en un entorno virtual viene dada por el grado en que responde de manera similar a como lo haría en la situación real que se está simulando. Entre los factores que influyen en la sensación de presencia se encuentran la inmersión y la interacción; es por ello que habitualmente se recurre a dispositivos y procedimientos que favorezcan altos niveles de estas dos variables. El uso de tecnología altamente interactiva e inmersiva a menudo conlleva una importante inversión económica y requiere que los profesionales adquieran una formación complementaria para su uso (Ferrer-García y Gutiérrez-Maldonado, 2012). Por otra parte, algunas personas informan de malestar durante y después de la exposición mediante RV inmersiva.
Es lo que se denomina cybersickness y tiene un amplio abanico de síntomas: visión borrosa, dolores de cabeza, náuseas, etc. (Gorini y Riva, 2008; Ferrer-García y Gutiérrez-Maldonado, 2012).

Aun con estas limitaciones, existe una larga lista de estudios que aportan evidencia sobre la utilidad de la RV para la evaluación y tratamiento psicológico, especialmente en el caso de las fobias y otros trastornos relacionados con la ansiedad (Powers y Emmelkamp, 2008; Meyerbröker y Emmelkamp, 2010). La exposición a estímulos mediante RV también se ha utilizado con éxito en el ámbito de las adicciones a sustancias, siendo capaz de provocar respuestas de craving en personas cuando son expuestas a situaciones o parafernalia relacionadas con su adicción (Bordnick et al., 2008; Ferrer-García et al., 2010; Ferrer-Garcia et al., 2012; Lee, Kwon, Choi, y Yang, 2007). Dadas las similitudes entre el deseo de consumir o craving experimentado por las personas con adicciones y con BN y TA, cabe esperar que el uso de la exposición a señales mediante realidad virtual pueda resultar un medio adecuado para el tratamiento de estas patologías. Por otro lado, la exposición a señales mediante RV también se ha mostrado eficaz para provocar ansiedad y otras emociones negativas y para provocar cambios en la imagen corporal de pacientes con TCA incluso empleando sistemas no inmersivos como la pantalla del ordenador (Ferrer-García et al., 2009; Gutiérrez-Maldonado et al., 2006; Gutiérrez-Maldonado et al., 2010).

El rápido avance de las tecnologías informáticas lleva a dispositivos cada vez más potentes pero también más económicos y de fácil manejo. Dentro de este último grupo, destacan los ordenadores con pantallas estereoscópicas que permiten la visión tridimensional de los entornos mediante el uso de gafas polarizadas. Este tipo de dispositivos ha sido poco empleado hasta el momento en los estudios realizados sobre aplicaciones de la RV en el ámbito de la psicología clínica, probablemente debido al menor grado de inmersión que permiten alcanzar, aunque requieren una inversión económica muy reducida y pueden emplearse por cualquier persona capaz de manejar un ordenador común. 
El objetivo de este estudio es evaluar si la exposición a alimentos virtuales mediante el uso de dispositivos de coste reducido, como son los ordenadores portátiles con pantalla estereoscópica, es capaz de producir niveles de craving consistentes con el craving-rasgo y con el craving-estado en una muestra de personas sin trastornos de la conducta alimentaria. Este indicador de validez de este tipo instrumentos sería un primer paso para su posible utilización en programas de tratamiento basados en exposición a señales virtuales para pacientes con trastornos alimentarios.

Se formula la hipótesis de que las personas que muestran mayores niveles de craving-rasgo y craving-estado, serán aquellas que también mostrarán mayores deseos de comer durante la exposición a alimentos virtuales en los diferentes entornos de RV.

\section{MÉTODO}

\section{Participantes}

La muestra está formada por 82 alumnos de grado de psicología de la Universidad de Barcelona, 66 mujeres y 16 hombres, sin trastornos de conducta alimentaria. El rango de edad va de los 20 a los 36 años, con una media de 22.57 y desviación típica de 3,06. El índice de masa corporal (IMC) medio es de $22.07(D T=3.07)$, con un rango desde 16,69 hasta 30.85 .

\section{Instrumentos}

Ordenadores portátiles estereoscópicos, auriculares y gafas polarizadas. Para la presentación de los entornos virtuales se utilizaron ordenadores portátiles Acer Aspire 5740DG, con los que es posible simular la visión estereoscópica mediante la utilización de filtros polarizadores. La interacción con los objetos podía realizarse con el propio teclado y panel táctil del dispositivo. Se utilizaron también auriculares canceladores de ruido.

Versión española del Food Craving Questionnaire-State (FCQ-S) (Cepeda-Benito et al., 2000). Mide la intensidad de los deseos de co- mer en el presente inmediato, mediante 15 ítems divididos en cinco dimensiones (Rodríguez, Mata, Moreno, Fernández y Vila, 2007).

Versión española del Food Craving Questionnaire-Trait (FCQ-T) (Cepeda-Benito et al., 2000). Mide la intensidad de los deseos de comer característicos de una persona mediante 37 ítems divididos en nueve dimensiones (Rodríguez, et al., 2007).

\section{Procedimiento}

Los participantes fueron evaluados en el laboratorio de RV de la Facultad de Psicología de la Universidad de Barcelona. Antes de iniciar la exposición a los entornos virtuales, los participantes fueron expuestos a la imagen 2D de 30 alimentos y se les pidió que indicasen el nivel de craving experimentado ante cada uno de ellos sobre una escala visual analógica de 0 a 100 puntos. A continuación se les presentó la imagen de cuatro contextos distintos (una cocina, un comedor, una cafetería y una habitación) y de nuevo se les pidió que indicaran el nivel de craving experimentado en cada uno de ellos sobre una escala visual analógica de 0 a 100 puntos. A partir de esta información, el programa construía una jerarquía de 40 entornos virtuales que resultaban de la combinación de los 10 alimentos del listado que provocaban más craving y de cada uno de los contextos evaluados. Esta jerarquía determinaba el orden de exposición ante las combinaciones de alimentos y entornos de cada participante, de manera que en primer lugar se le exponía al alimento asociado con menor craving en el contexto asociado con menor craving; a continuación se le exponía al alimento asociado con menor craving en el contexto siguiente, y así sucesivamente hasta pasar por los cuatro contextos. Esta secuencia se repetía de la misma forma con los siguientes alimentos de la jerarquía.

Una vez dentro del entorno virtual, se pedía a los participantes que buscasen su mesa y se sentasen en una de las sillas. Sobre la mesa se encontraba el alimento correspondiente según la jerarquía. Usando el ratón del ordenador, el participante podía levantar el plato con el ali- 


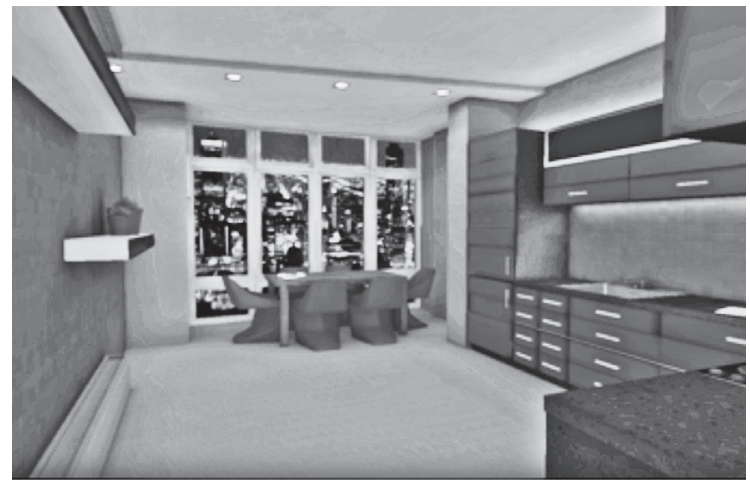

Cocina

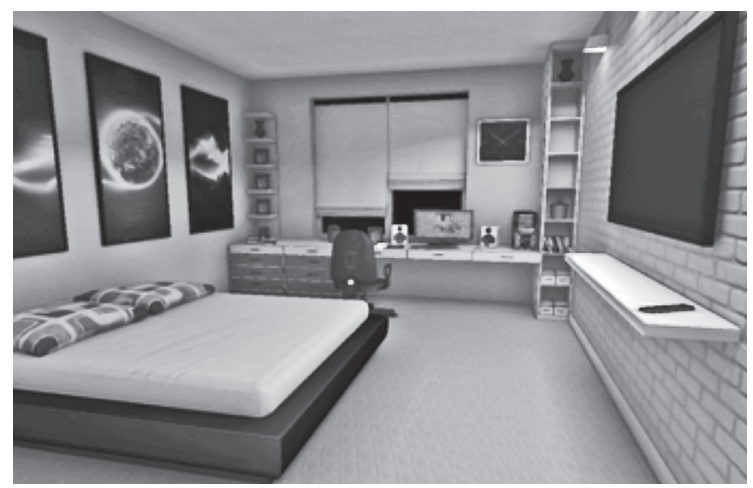

Dormitorio

Figura 1. Imágenes de los entornos virtuales

mento, girarlo y acercárselo. Tras 20 segundos sentado a la mesa y manipulando el alimento de esta manera, se evaluaba el nivel de craving mediante una escala visual analógica de 0 a 100 puntos. Una vez respondida la pregunta se pasaba al siguiente nivel de la jerarquía hasta completar los 40 entornos. Una vez finalizada la exposición a los entornos virtuales, los participantes rellenaron los cuestionarios FCQ-T y FCQ-S.

\section{Análisis estadísticos}

Se llevaron a cabo análisis correlacionales para evaluar la asociación entre el craving-rasgo y el craving-estado evaluados con los cuestionarios de lápiz y papel y el craving medio generado por la exposición a los 10 alimentos en cada uno de los entornos de realidad virtual. Se realizaron también diversos análisis de re-

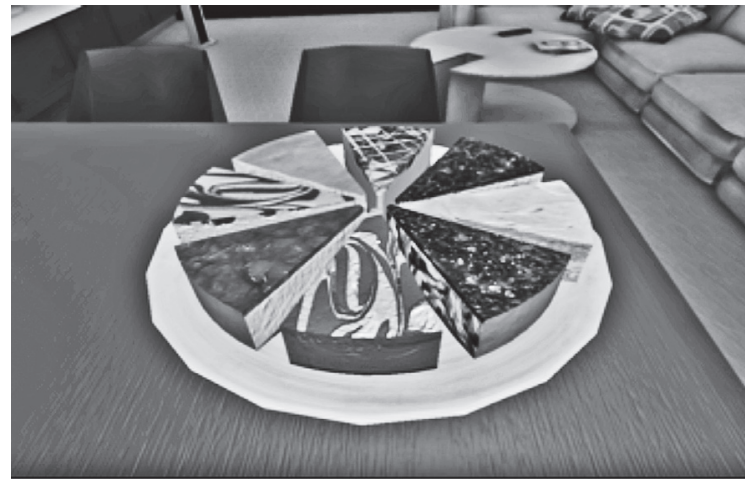

Comedor

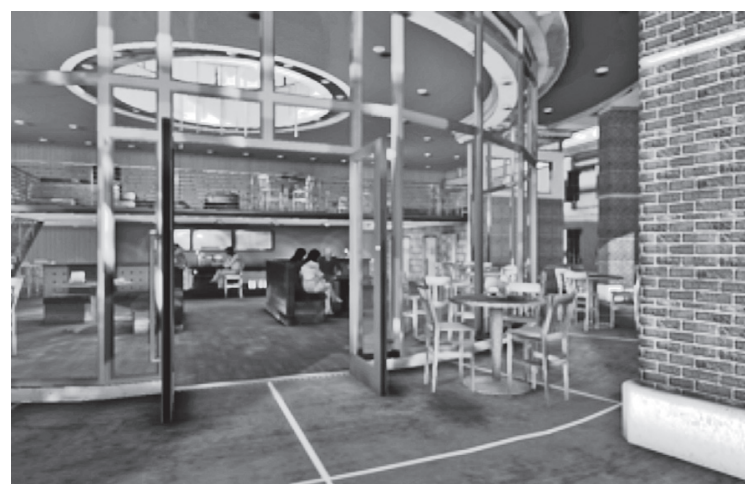

Cafetería

gresión múltiple para evaluar qué porcentaje de la variancia de las respuestas de craving en los diferentes entornos virtuales era explicado por las puntuaciones obtenidas en los dos cuestionarios, así como para evaluar cuál de las dos variables incluidas en el modelo (el craving-rasgo o el craving-estado) contribuía en mayor medida a la predicción del craving experimentado en los entornos de realidad virtual. El análisis de datos se llevó a cabo con el programa estadístico SPSS v.20.

\section{RESULTADOS}

Tal como se muestra en la Tabla 1, las correlaciones entre las puntuaciones obtenidas en el FCQ-T, el FCQ-S y el craving medio experimentado en cada uno de los cuatro entornos virtuales son positivas y significativas, con una excepción: el craving-rasgo de los participantes 
no se relaciona significativamente con el deseo de comer reportado en el comedor virtual.

Tabla 1. Correlaciones entre la puntuación total de los cuestionarios FCQ-T y FCQ-S y los niveles medios de deseo de comer experimentados en cada uno de los entornos virtuales evaluados.

\begin{tabular}{lcccc}
\hline & Cocina & Comedor & Dormitorio & Cafetería \\
\hline FCQ-T & $0,252^{*}$ & 0,173 & $0,284^{*}$ & $0,236^{*}$ \\
FCQ-S & $0,296^{* *}$ & $0,227^{*}$ & $0,303^{* *}$ & $0,232^{*}$ \\
\hline Nota. ${ }^{*} p<.05 ; * *<.01$. & &
\end{tabular}

Los resultados de los análisis de regresión múltiple se presentan en la Tabla 2. El modelo formado por las variables craving-rasgo y craving-estado es capaz de predecir el craving experimentado en la cocina, el dormitorio y la cafetería virtuales. Sin embargo, es necesario resaltar que el nivel de varianza explicado por el modelo es pequeño, fluctuando entre el 8 y el $13 \%$. El craving-estado es la variable que hace la mayor contribución única a la predicción del craving en todos los entornos virtuales menos en la cafetería, donde el craving-rasgo hace una contribución mayor. En la cocina $\mathrm{y}$ en el dormitorio la contribución única del craving-estado llega a ser estadísticamente significativa ( $p=0,043$ y $p=0,045$ respectivamente).

Finalmente, se llevó a cabo otro análisis de regresión múltiple, utilizando el craving medio de los sujetos en los cuatro entornos como variable dependiente, y las puntuaciones de los test FCQ-S y FCQ-T como variables predictoras. Los resultados del análisis (Tabla 2) muestran que el modelo explica el $10 \%$ de la varianza del craving medio experimentado en el conjunto de entornos de RV. De nuevo, las puntuaciones de craving-estado medidas con el FCQ-S (Beta=0,213) son más efectivas que las puntuaciones del craving-rasgo medidas con el FCQ-T $($ Beta $=0,168)$ para predecir el craving que genera la exposición a alimentos en los entornos virtuales.

\section{DISCUSIÓN}

De acuerdo con lo esperado, las personas que presentan mayores niveles de craving-rasgo y estado, son aquellas que también muestran mayores deseos de comer durante la exposición a los diferentes entornos de RV. Además, el craving-estado y rasgo, evaluados con el FCQ-

Tabla 2. Análisis de regresión en los que las puntuaciones obtenidas en los cuestionarios FCQ-T y FCQ-S predicen el deseo de comer medio experimentado en cada uno de los entornos de realidad virtual

\begin{tabular}{|c|c|c|c|c|c|c|c|c|}
\hline Modelo & Predictores & Beta & $t$ & $p$ & $\mathrm{R}^{2}$ & $\mathrm{R}^{2}$ adj. & $F$ & $p$ \\
\hline \multirow[t]{3}{*}{ Cocina } & & & & & .112 & .089 & 4.91 & .010 \\
\hline & FCQ-T & .168 & 1.466 & .147 & & & & \\
\hline & FCQ-S & .235 & 2.058 & .043 & & & & \\
\hline \multirow[t]{3}{*}{ Comedor } & & & & & .061 & .037 & 2.537 & .086 \\
\hline & FCQ-T & .105 & .896 & .373 & & & & \\
\hline & FCQ-S & .189 & 1.605 & .112 & & & & \\
\hline \multirow[t]{3}{*}{ Dormitorio } & & & & & .127 & .105 & 5.673 & .005 \\
\hline & FCQ-T & .200 & 1.767 & .081 & & & & \\
\hline & FCQ-S & .231 & 2.040 & .045 & & & & \\
\hline \multirow[t]{3}{*}{ Cafetería } & & & & & .081 & .057 & 3.419 & .038 \\
\hline & FCQ-T & .175 & 1.506 & .136 & & & & \\
\hline & FCQ-S & .169 & 1.453 & .150 & & & & \\
\hline \multirow[t]{3}{*}{ Total } & & & & & .099 & .076 & 4.302 & .017 \\
\hline & FCQ-T & .168 & 1.455 & .150 & & & & \\
\hline & FCQ-S & .213 & 1.852 & .068 & & & & \\
\hline
\end{tabular}


$\mathrm{S} / \mathrm{T}$, son capaces de predecir el craving medio experimentado en la cocina, el dormitorio y la cafetería. A pesar de ello, el hecho de que el porcentaje de varianza explicado sea bajo indica que hay otros aspectos implicados en la aparición del deseo de comer que deben ser establecidos y tomados en consideración. El estado de ánimo, la restricción alimentaria, el estado motivacional hacia la comida (apetitivo versus defensivo) o la mayor o menor sensibilidad a la recompensa, entre otras, se han propuesto como variables que modularían la respuesta de craving ante la comida (Gómez et al., 2011; Moreno, Rodríguez y Fernández, 2009; Tetley, Brunston, y Griffiths, 2009).

En general, el craving-estado, evaluado con el FCQ-S, parece ser mejor predictor del $\mathrm{cra}^{-}$ ving provocado por la exposición a alimentos en los entornos de realidad virtual que el craving-rasgo (FCQ-T). Estudios previos que también han empleado el FCQ-T/S señalan que las personas que muestran mayores niveles de $\mathrm{cra}$ ving-rasgo son aquellas que realizan más atracones y presentan más tendencia a perder el control sobre la ingesta (Gómez et al., 2011). Por tanto, sería esperable que en población clínica la puntuación obtenida en el FCQ-T resultase mejor predictor que el FCQ-S. Sin embargo, dado que la muestra en este estudio está formada por estudiantes universitarios sin TCA, es posible que aspectos más contextuales, y no tanto variables relacionadas con diferencias individuales, estén influyendo en la respuesta que dan ante los entornos. El tiempo transcurrido desde la última comida, por ejemplo, es una variable que no se controló en este estudio y que pudo influir en los resultados. Otra variable que pudo afectar a los resultados, y que no se ha controlado en este experimento, es el posible seguimiento de dietas. Según el estudio realizado por Pelchat y colaboradores (Pelchat, Johnson, Chan, Valdez, y Ragland, 2004) el craving ante los alimentos es más intenso y se da con más frecuencia en las personas que siguen una dieta que en aquellas que no lo hacen $\mathrm{y}$, además, es independiente del hambre.

Otro punto a considerar es que los participantes sólo se han expuesto a los 10 alimentos del listado inicial de 30 que más puntuación en craving han alcanzado. Esto implica que cada participante puede haberse expuesto a platos de comida diferentes. Perpiñá et al. (2013) encontraron que la presencia de alimentos altamente calóricos y relacionados con el incremento de peso (por ejemplo, pizza, helado o dulces) produce respuestas distintas en personas con TCA y personas sin esta patología, mostrando las personas diagnosticadas más miedo y más evitación. Aunque el presente estudio solo incluye participantes sin TCA, es necesario tener en cuenta que la exposición a diferentes alimentos puede haber influido en las respuestas dadas por cada individuo.

Aunque con las consideraciones y limitaciones expuestas, puede concluirse que la exposición a comida en entornos de RV no inmersiva mediante el uso de dispositivos de bajo coste y fácil uso como son los ordenadores portátiles estereoscópicos es un procedimiento válido para llevar a cabo intervenciones basadas en técnicas de exposición a señales. Como se había previsto, los niveles de craving experimentados durante las exposiciones virtuales guardan una relación coherente con los niveles de $\mathrm{cra}$ ving-rasgo y craving-estado de los participantes.

\section{REFERENCIAS}

American Psychiatric Association (2013). Diagnostic and Statistical Manual of Mental Disorders, Fifth Edition. Arlington, VA.

Bordnick, P.S., Traylor, A., Copp, H.L., Graap, K.M., Carter, B., Ferrer, M., \& Walton, A. P. (2008). Assessing reactivity to virtual reality alcohol based cues. Addictive Behaviors, 33, 743-756.

Botella, C., García-Palacios, A., Baños, R. M., y Quero, S. (2007). Realidad virtual y tratamientos psicológicos. Cuadernos de Medicina Psicosomática y Psiquiatría de Enlace, 82, 17-31.

Cepeda-Benito, A., Gleaves, D.H., Fernández, M.C., Vila, J., Williams, T., \& Reynoso, J. (2000). The development and validation of Spanish version of the State and Trait Food Cravings Questionnaires. Behaviour Research and Therapy, 38, 1125-1138. doi: 10.1016/S00057967(99)00141-2.

Ferrer-García, M., García-Rodríguez, O., GutiérrezMaldonado, J., Pericot-Valverde, I., \& Secades-Villa, R. (2010). Efficacy of virtual reality for triggering smoking craving: relation with level of presence and nicotine dependence. Studies in Health Technology and Informatics, 154, 123-127. 
Ferrer-García, M., García-Rodríguez, O., Pericot-Valverde, I., Yoon, J.H., Secades-Villa, R., \& GutiérrezMaldonado, J. (2012). Predictors of smoking craving during virtual reality exposure. Presence: Teleoperators and Virtual Environments, 21, 423-434.

Ferrer-García, M., \& Gutiérrez-Maldonado, J. (2012). The use of virtual reality in the study, assessment, and treatment of body image in eating disorders and nonclinical samples: A review of the literature. Body Image, 9, 1-11.

Ferrer-García, M., Gutiérrez-Maldonado, J., CaqueoUrízar, A., \& Moreno, E. (2009). The validity of virtual environments for eliciting emotional responses in patients with eating disorders and in controls. Behavior Modification, 33, 830-854. doi : $10.1177 / 0145445509348056$.

García-Rodríguez, O., Pericot-Valverde, I., GutiérrezMaldonado, J., y Ferrer-García, M. (2009). La realidad virtual como estrategia para la mejora de los tratamientos del tabaquismo. Health and addictions/ Salud y drogas, 9, 39-55.

Gómez, M.A., Yela, J.R., Salgado, A., y Cortés, M. (2011). Evaluación de la reactividad emocional ante imágenes de comida en bulimia nerviosa. Psicothema, 23, 580-586.

Gorini, A., \& Riva, G. (2008). Virtual reality in anxiety disorders: The past and the future. Expert Review of Neurotherapeutics, 8, 215-233. doi: 10.1586/14737175.8.2.215.

Gutiérrez-Maldonado, J. (2002). Aplicaciones de la realidad virtual en psicología clínica. Aula médica psiquiatría, 4, 92-126.

Gutiérrez-Maldonado, J., Ferrer-García, M., CaqueoUrízar, A., \& Letosa-Porta, A. (2006). Assessment of emotional reactivity produced by exposure to virtual environments in patients with eating disorders. Cyberpsychology \& Behavior, 9, 507-513.

Gutiérrez-Maldonado, J., Ferrer-García, M., CaqueoUrízar, A. \& Moreno, E. (2010). Body image in eating disorders: The influence of exposure to virtual-reality environments. Cyberpsychology, Behavior, and Social Networking, 13, 521-531. doi: 10.1089/cyber.2009.0301

Gutiérrez-Maldonado, J., Ferrer-García, M., Riva, G. (2013). VR cue-exposure treatment for bulimia nervosa. Studies in Health Technology and Informatics, 191, 21-25.

Jansen, A. (1994). The learned nature of binge eating, In C.R. Legg and D.A. Booth (Eds.), Appetite: Neural and behvioural bases, 193-211. Oxford University Press: Oxford.

Jansen, A. (1998). A learning model of binge eating: Cue reactivity and cue exposure, Behaviour Reasearch and Therapy, 36, 257-272.

Jansen, A., Broekmate, J., \& Heymans, M. (1992). Cue exposure vs. self control in the treatment of binge eating. Behaviour Research and Therapy, 3, 235-241.
Jansen, A., van den Hout, M.A., de Loof, C., Zandbergen, J., \& Griez, E. (1989). A case of bulimia successfully treated by cue exposure. Journal of Behavior Therapy and Experimental Psychiatry, 20, 327-332.

Koskina, A., Campbell, I., \& Schmidt, U. (2013). Exposure therapy in eating disorders revisited. Neuroscience and Behavioral Reviews, 37, 193-208. doi: 10.1016/j. neubiorev.2012.11.010.

Lee, J.H., Kwon, H., Choi, J., \& Yang, B.H. (2007). Cueexposure therapy to decrease alcohol craving in virtual environment. Cyberpsychology \& Behavior, 10, 617623. doi: 10.1089/cpb.2007.9978.

Martínez-Mallén, E., Castro, J., Lazaro, L., Moreno, E., Morer, A., Font, E., Julien, J., Vila, M., \& Toro, J. (2007). Cue exposure in the treatment of resistant adolescent bulimia nervosa. International Journal of Eating Disorders, 40, 596-601.

Meyerbröker, K., \& Emmelkamp, P.M. (2010). Virtual reality exposure therapy in anxiety disorders: A systematic review of process-and-outcome studies. Depression and Anxiety, 27, 933-944. doi: 10.1002/ da.20734.

Moreno S., Rodríguez S., y Fernández, M.C. (2009). ¿Qué es el ansia por la comida? Madrid: Pirámide.

Nederkoon, C., \& Jansen, A. (2002). Cue-reactivity and regulation of food intake. Eating Behavior, 3, 61-72.

Pallavicini, F., Cipresso, P., Raspelli, S., Grassi, A., Serino, S., Vigna, C., Triberti, S., Villamira, M., Gaggioli, A., \& Riva, G. (2013). Is virtual reality always an effective stressors for exposure treatments? Some insights from a controlled trial. BMC Psychiatry, 13(52), 1-11. doi: 10.1186/1471-244X-13-52.

Pelchat, M.L., Johnson, A., Chan, R., Valdez, J., \& Ragland, J.D. (2004). Images of desire: food-craving activation during fMRI. Neuroimage, 23, 1486-1493.

Perpiñá, C., Roncero, M., Fernández-Aranda, F., JiménezMurcia, S., Forcano, L., \& Sánchez, I. (2013). Clinical validation of a virtual environment for normalizing eating patterns in eating disorders. Comprehensive Psychiatry, 54, 680-686. doi: 10.1016/j.comppsych.2013.01.007.

Powers, M.B., \& Emmelkamp, P.M.G. (2008). Virtual reality exposure therapy for anxiety disorders: A metaanalysis. Journal of Anxiety Disorders, 22, 561-569.

Rodríguez, S., Mata, J.L., y Moreno, S. (2007). Psicofisiología del ansia por la comida y la bulimia nerviosa. Clínica y Salud, 18, 99-118.

Rodríguez, S., Mata, J.L., Moreno, S., Fernández, M.C., y Vila, J. (2007). Mecanismos psicofisiológicos implicados en la regulación afectiva y la restricción alimentaria de mujeres con riesgo de padecer bulimia nerviosa. Psicothema, 19, 30-36.

Schmidt, U., \& Marks, I.M. (1988). Cue exposure to food plus response prevention of binges for bulimia: A pilot study. International Journal of Eating Disorders, 7, 663-672. 
Tetley, A., Brunstrom, J., \& Griffiths, P. (2009). Individual differences in food-cue reactivity. The role of BMI and everyday portion-size selections. Appetite, 52, 614620 .
Toro, J., Cervera, M., Feliu, M.H., Garriga, N., Jou, M., Martinez, E. , \& Toro, E. (2003). Cue exposure in the treatment of resistant bulimia nervosa. International Journal of Eating Disorders, 34, 1-8. 
